
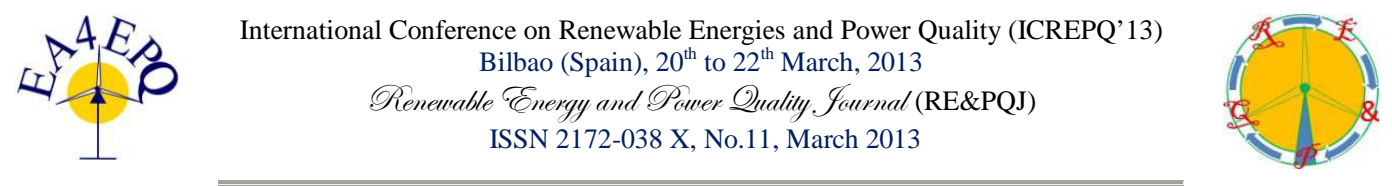

\title{
Optimization of efficiency and energy saving in public lighting with multi-objective evolutionary algorithms
}

\author{
D. Gómez-Lorente, O. Rabaza, A. Espín, A. Peña-García \\ Department of Civil Engineering \\ E.T.S.I.C.C.P., University of Granada \\ Campus Fuentenueva - 18071 Granada (Spain)
}

Phone/Fax number: +0034 958249 435, e-mail: dglorente@ugr.es, ovidio@ugr.es, aespin@ugr.es, pgarcia@ugr.es.

\begin{abstract}
Multi-objective evolutionary algorithms have been applied to classical problems in public lighting calculations with the aim of finding the most optimal solutions among the very large set of different configurations achieved with the standard methods.
\end{abstract}

\section{Key words}

Installation efficiency, Numerical optimization, Roadway lighting.

\section{Introduction}

Although the absolute need of public lighting to ensure the safety of people and goods is nowadays out of doubt, its economic and environmental impact of public lighting is huge. Hence, it is frequent to find streets, roads or tunnels with remarkably high illumination levels.

However, in spite of these high levels, accurate visual comfort and performance are not always achieved because of the lack of uniformity or the glare caused by the lighting installations.

The remarks above highlight the necessity of optimizing the public lighting installations in order to provide optimal visual performance and spare energy.

In this communication, we propose an effective and efficient multi-objective evolutionary algorithm (MOEA) called NSGA-II that incorporates specific mechanisms in order to better tackle the roadway lighting optimization problem by using the following two objectives:

- Maximizing the overall illuminance uniformity, $U_{0}$.

- Maximizing the installation efficiency, $\varepsilon$.

These parameters are defined as:

$$
U_{0}=\frac{E_{\min }}{E_{a v}}
$$

where $E_{\min }$ is the minimum value of the lluminance values over all units between the next two lighting fittings and $E_{a v}$ is the average illuminance, and [1],

$$
\mathcal{E}=\frac{A_{T} E_{a v}}{P_{T}}
$$

where $A_{T}$ is the illuminated surface, $E_{a v}$ is the average illuminance on the ground and $P_{T}$ is the total electrical power installed, including the light sources and the electrical auxiliary devices. This parameter can also be expressed as a function of individual rectangles influenced by one given luminary as follows:

$$
\varepsilon=\frac{N A \cdot E_{a v}}{N P}=\frac{A \cdot E_{a v}}{P}
$$

where $A$ is the surface of one individual rectangle, $E_{a v}$ is the average illuminance on the ground and $P$ is the electrical power consumed by one individual luminary including its light sources and electrical auxiliary devices.

In the considered configurations, the algorithm chooses and distributes the elements that make up the public lighting such as, the height of the lamps and the spacing between them in such a way as to maximize the two above objectives. Both elements will be part of the solution of the problem, which will allow us to know the overall illuminance uniformity and the installation efficiency. The experimental study will include a comparison of the results obtained by NSGA-II with as well-known and reliable free software. 
The rest of the paper is organized as follows: Section 2 we present the use of NGSA-II for optimizing the road lighting. Section 3 discusses roads lighting criteria and presents the analysis of results. Finally, in Section 4 we summarize our conclusions..

\section{NSGA-II optimization process}

A MOEA, known as the Non-dominated Sorting Genetic Algorithm II (NSGA-II) [2] was used to study the overall uniformity and installation efficiency of the roadway lighting, which are the two optimization variables. Nondominated sorting in genetic algorithms is a popular nondomination based genetic algorithm for multi-objective optimization [3]. In this paper, the new version, NSGA-II, which has a better sorting algorithm, is applied. This algorithm is similar to a conventional genetic algorithm involving the following steps: population initialization, fitness evaluation, reproduction (parent selection), simulated binary recombination crossover [4], and mutation. The main difference between NSGA and NSGAII is the inclusion of the non-dominated sort classification and crowding distance.

These objectives are contradictory. In fact, as the overall uniformity increases, so does the spacing between the streetlights. This means that, the installed power in the street will be greater, and thus, the installation will be less efficient. Consequently, the bi-objective optimization outcomes are a solution set that is a compromise between these two objectives. The genetic algorithm parameters used for the different optimizations are shown in Table I.

\begin{tabular}{lr}
\hline Parameter & Value \\
\hline Number of generations (Ngen) & 150 \\
Number of individuals per generation & 100 \\
Crossing probability & $90 \%$ \\
Mutation probability & $10 \%$ \\
\hline
\end{tabular}

Table I. NGSA-II parameters used for the optimization

In this case, the street width and lamp type are input parameters. Our objective was to evaluate the influence of the location and characteristics of the luminaires on the performance of the roadway lighting system.

\section{Materials and Methods}

A free software frequently used for lighting calculations called DIALUX has been first used as a classical way of calculation to be compare later with the results achieved with the proposed method.

Then, the average illuminance, $E_{a v}$ has been calculated by means of its classical definitions, that is, for one given parameterized luminary [5], defined by a matrix of intensities in the directions of the angles $C$ and $\gamma$ :

$$
E_{a v}=\frac{1}{N} \sum \frac{I(C, \gamma)}{h^{2}} \cdot \cos ^{3} \gamma
$$

where $I(C, \gamma)$ is the matrix of intensities, $h$ is the mounting height of the luminary, $N$ the number of points considered for the average (as high as possible).

The overall illuminance uniformity, $U_{0}$, given by equation (1) and the efficiency of the installation, given by (2), have been also calculated. The average illuminance and the overall illuminance uniformity given by these equations have been compared with the results of DIALUX in several configurations with identical results (see Table II):

\begin{tabular}{|l|c|c|c|c|c|c|}
\hline \multirow{2}{*}{ Config. } & \multicolumn{2}{|c|}{ One Sided } & \multicolumn{2}{c|}{$\begin{array}{c}\text { Two Sided } \\
\text { Staggered }\end{array}$} & \multicolumn{2}{c|}{ Two Sided Coupled } \\
\cline { 2 - 7 } & Equat. & DIAL & Equat. & DIAL & Equat. & DIAL \\
\hline$E_{a v}(\operatorname{lux})$ & 15.65 & 15.00 & 16.33 & 16.00 & 16.33 & 16.00 \\
\hline$U_{0}$ & 0.40 & 0.40 & 0.53 & 0.50 & 0.18 & 0.20 \\
\hline$\varepsilon$ & 35.94 & 34.44 & 35.00 & 34.30 & 32.82 & 36.73 \\
\hline
\end{tabular}

Table II. Comparison between direct evaluation with equations (1) and (4) and DIALUX

Table II shows that the results are very similar. This allows us to directly work with equations (1) and (4) instead of DIALUX and thus generate a much bigger set of solutions (between 85 and 90) to be optimized later with the multi-objective evolutionary algorithm.

The following step has been to optimize the set of results generated in terms of efficiency from given values of uniformity. The application of the (MOEA) have given results as those shown in Figure 1, which shows one typical set of solutions for one given configuration (one sided) and light source (High Pressure Sodium). Each solution includes spacing between luminaries, height of the luminaries, overall illuminance uniformity and efficiency of the installation.

\section{ONE SIDED HPS 70W}

$\begin{array}{cccc}\text { SPACING } & \text { HEIGHT } & \text { UNIFORMITY EFFICIENCY } \\ 17,1553872 & 5,00000000 & 0,21410273 & 43,557971 \\ 17,1504285 & 5,89875626 & 0,39073599 & 41,3463993 \\ 15,0650297 & 5,59496583 & 0,34598442 & 41,3692066 \\ 16,2282196 & 6,24838512 & 0,51748134 & 40,1464764 \\ 16,2598751 & 6,39406627 & 0,56118726 & 39,5386916 \\ 16,2195791 & 6,0637876 & 0,46255105 & 40,7231717 \\ 17,146243 & 6,03393113 & 0,43397714 & 41,000501 \\ 16,2771633 & 6,14326988 & 0,48744898 & 40,1724548 \\ 16,2218699 & 5,91065216 & 0,413455 & 41,1271349 \\ 17,1542677 & 6,07958219 & 0,44647889 & 40,8281979 \\ 18,1869377 & 5,52689444 & 0,28001164 & 42,1279389 \\ \ldots & \ldots & \ldots & \ldots\end{array}$

Figure 1. Sets of solutions for one given configuration and light source. 
The plots of these results for each configuration and light source are shown in Figures 2, 3 and 4:

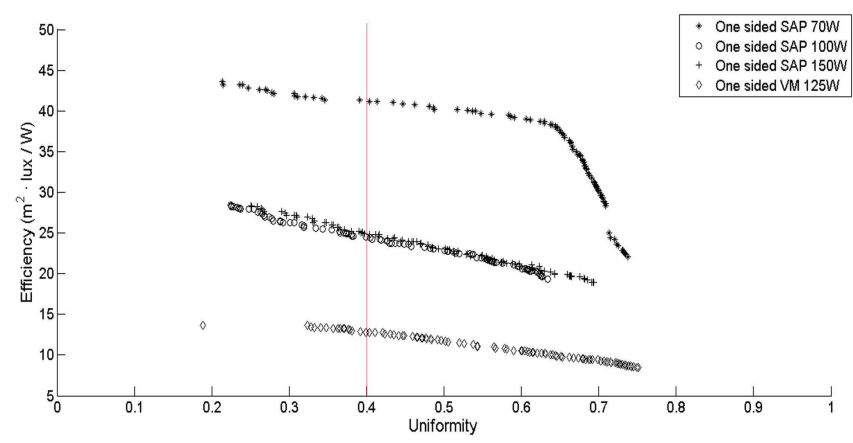

Figure 2. Efficiency of the solutions as a function of overall illuminance uniformity in a one sided configuration. HPS means high pressure sodium and HPM, high pressure mercury.

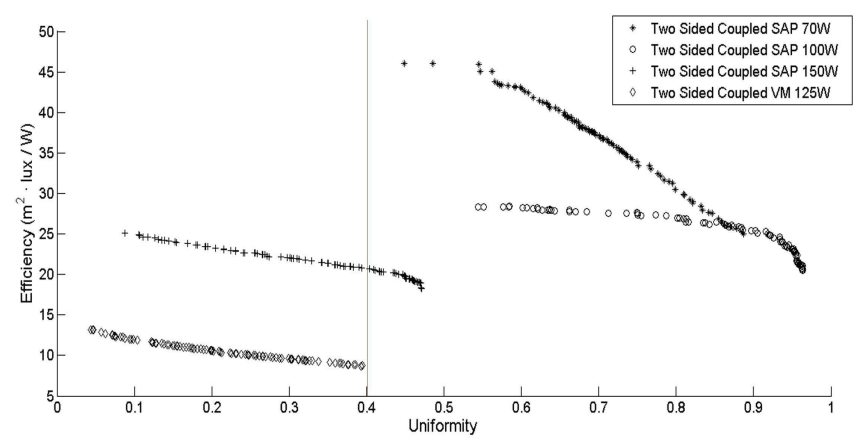

Figure 3. Efficiency of the solutions as a function of overall illuminance uniformity in a two sided coupled configuration. HPS means high pressure sodium and HPM, high pressure mercury.

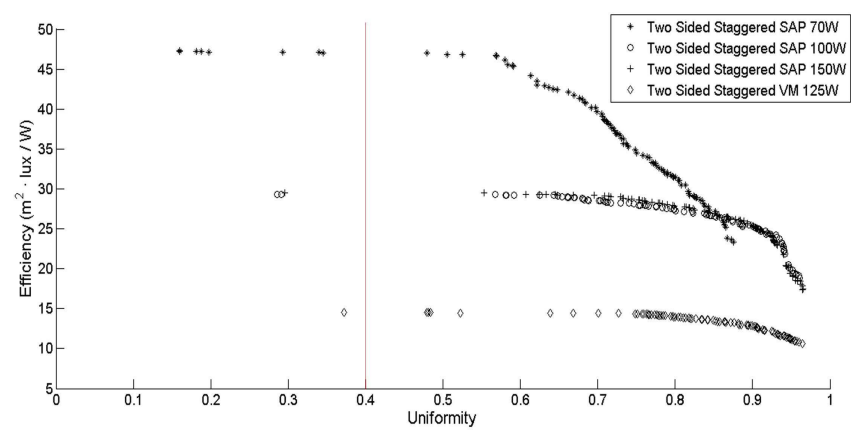

Figure 4. Efficiency of the solutions as a function of overall illuminance uniformity in a two sided staggered configuration. HPS means high pressure sodium and HPM, high pressure mercury.

The figures above show that there is a linear relationship between the installation efficiency, $\mathcal{E}$, and the overall illuminance uniformity, $U_{0}$. Given that equation (3) provides a relationship between efficiency and the geometrical parameters of the installation that can also be expressed as follows:

$$
\varepsilon=\frac{l \cdot w \cdot E_{a v}}{P}
$$

where $l$ is the length of one element of street illuminated by one single luminary and $w$ the width of the street $(w / 2$ if we work with a two sided coupled configuration), we can choose one point whose efficiency is maximal for a given uniformity (it is not possible with the current software), and calculate the main parameters of the installation like spacing between luminaries.

This is the great advantage of the method. The software commonly used for lighting design does not allow to maximize $\mathcal{E}$ for the uniformity we choose and, for this reason, some efficient installations sometimes have bad visual performances due to the lack of uniformity.

\section{Conclusion}

Several important conclusions can be taken from the results above:

1) The important role of efficiency in public lighting makes necessary to consider this parameter as a main output in all the software and methods currently used to design such installations. This importance comes from the fact that efficiency informs us about how economical and environmental friendly one installation is.

2) The use of multi-objective evolutionary algorithms (MOEAs), allows the achievement of optimal results in terms of both, overall illuminance uniformity, $U_{0}$, and installation efficiency, $\varepsilon$.

3) The configuration of the lighting installation (one sided, two sided coupled or two sided staggered) has a big impact on efficiency. Thus, according to the results reported in this research, the two-sided staggered installation yields the best results in terms in efficiency among the three configurations simulated for one given street with fix conditions of mean illuminance.

4) In addition to these results, the optimization with a multi-objective evolutionary algorithm of the different possible solutions to the computational simulation of a public lighting installation, yields a set of solutions in terms of efficiency, overall illuminance uniformity, height of luminaries and spacing, whose mutual relationships are purely linear or composed by two well defined lines. 
5) These relationships are an extremely useful tool to easily calculate the main parameters in public lighting installations. This may provide a quick method to design sustainable installations even without computational tools, which could be really interesting in cooperation with not developed countries.

\section{References}

[1] Real Decreto 1890/2008 (Reglamento de eficiencia energética en instalaciones de alumbrado exterior y sus Instrucciones técnicas complementarias EA-01 a EA-07). Ministerio de Industria, Turismo y Comercio de España. 2008.

[2] Deb, K., Pratap, A., Agarwal, S., \& Meyarivan, T. (2002). A Fast and Elitist Multiobjetive Genetic Algorithm: NSGA-II.

IEEE Transactions on Evolutionary Computation, 6(2), 182-197.

[3] Srinivas, N., \& Deb, K. (1994). Multiobjective optimization using nondominated sorting in genetic algorithms. IEEE Transactions on Evolutionary Computation, 2(3), 221-248.

[4] Deb, K., \& Agrawal, R. B. (1995). Simulated binary crossover for continuous search space. Complex Systems, 9(2), $115-148$.

[5] CIE (International Commission on Illumination) (2010). Lighting of Roads for Motor and Pedestrian Traffic, CIE Public 115, Vienna, Austria. 